\title{
O primeiro checklist de Angiospermas do Município de Nova Bandeirantes, Mato Grosso, Brasil
}

\author{
Ricardo da Silva Ribeiro ${ }^{1} \mathbb{1}^{1,3^{*}}$ Nhaára Da Vila Pereira $\mathbb{( 1}^{2}$ Karen Ribeiro Cruz ${ }^{3} \mathbb{C}^{1}$ Anderson Alex \\ Sandro Domingos de Almeida $\mathbb{1}^{4}$ Sara Mineli Caioni Cardoso $\mathbb{C}^{3}$ Rariane da Silva Ribeiro $\mathbb{1}^{5}$ Célia \\ Regina Araújo Soares-Lopes $\mathbb{1}^{3}$
}

\footnotetext{
${ }^{1}$ Instituto Nacional da Mata Atlântica, Av. José Ruschi, 4, CEP 29650-000, Santa Teresa, ES, Brasil

${ }^{2}$ Universidade Federal do Mato Grosso, Av. Fernando Corrêa da Costa, 2367, Boa Esperança, CEP 78060-900, Cuiabá, MT, Brasil

${ }^{3}$ Universidade do Estado de Mato Grosso, Campus I, Herbário da Amazônia Meridional, MT - 208, CEP 78580-000, Alta Floresta, MT, Brasil

${ }^{4}$ Universidade do Estado de Mato Grosso, Campus II, Laboratório de Ecologia Vegetal, Campus Av. Perimetral Rogério Silva - Norte 2, CEP 78580-000, Alta Floresta, MT, Brasil

${ }^{5}$ Universidade do Estado de Mato Grosso, Campus II, Faculdade de Direito, Campus II- Alta Floresta, CEP 78580-000, Alta Floresta, MT, Brasil
}

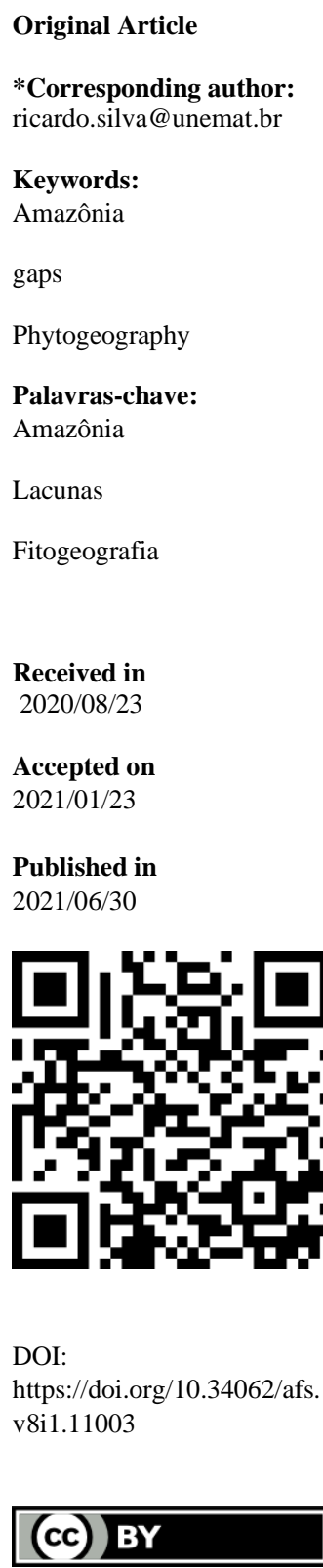

DOI:

https://doi.org/10.34062/afs. v8i1.11003

\section{(c) $\mathbf{B Y}$}

RESUMO: As listas taxonômicas são ferramentas importantes para conhecimento e conservação da biodiversidade. Aqui neste estudo, com base em expedições de campo e análise de bancos de dados disponíveis online de coleções biológicas, propomos: (i) a primeira lista de verificação de plantas vasculares (Angiospermas) para o município de Nova Bandeirantes, MT, Brasil e discutimos (ii) fitogeografia, lacunas e avanços no conhecimento da flora local. Com 399 registros, 153 espécies, 88 gêneros e 48 famílias, nossas análises inferem: (i) alta riqueza de Bignoniaceae, Fabaceae, Moraceae e Rubiaceae. (ii) baixa densidade de coletas, c.a. de 0,02 registro $/ \mathrm{km}^{2}$ e (iii) 20 padrões fitogeográficos, com predominância de espécies Amazônicas (68\%), além de apresentar 29 novas ocorrências em Mato Grosso. Esse estudo serve como base para uma sequência de trabalhos com objetivos de apresentar uma lista da flora de cada município no estado de Mato Grosso.

\section{The first checklist of Angiosperms of Nova Bandeirantes municipality, Mato Grosso, Brazil}

ABSTRACT: Taxonomic lists are important tools for knowledge and conservation of biodiversity. Here in this study, based on field expeditions and analysis of available online databases of biological collections, we propose: (i) the first checklist of vascular plants (Angiosperms) for the municipality of Nova Bandeirantes, MT, Brazil and we discuss (ii) phytogeography, gaps and advances in knowledge of the local flora. With 399 records, 153 species, 88 genera and 48 families. Our inferred analyzes: (i) high richness of Bignonicaeae, Fabaceae, Moraceae and Rubiaceae, (ii) low density of botanicals records $\left(0,02\right.$ records $\left./ \mathrm{km}^{2}\right)$ and (iii) 20 phytogeographic patterns, with predominance of Amazonian species. These studies also present new occurrences for the state of Mato Grosso. It serves as a pilot for a sequence of works with the purpose of presenting a list of the flora of each municipality in the state of Mato Grosso. 
Introdução

Checklists (listas de verificação de espécies) são importantes ferramentas para análise do conhecimento da biodiversidade e são extremamente dinâmicas, principalmente na Amazônia (Campbell 1989; Bicudo 2004; Thomas et al. 2012; Cardoso et al. 2017; Ter Stegge 2019). As listas de espécies podem ter caráter local, regional (Zappi et al. 2011; Lopes et al. 2014; Zappi et al. 2016; Sores-Lopes et al. 2016b), ou geopolítico municipal (Almeida 2018; Cardoso 2019), estadual (Dutra et al. 2015), ou para um país (Forzza et al. 2010; BFG 2015; 2018). Também podem apresentar a representatividade de coleções biológicas (Lanna et al. 2018) e/ou visando grupos taxonômicos (Fernandes et al. 2015; Almeida et al. 2016; Ribeiro et al. 2016; Teixeira et al. 2016; Silva et al. 2015; Ribeiro 2018; Ribeiro et al. $2018 \mathrm{a} ; \mathrm{b} ; \mathrm{c})$. Independente da escala ou o foco que se dão, os objetivos dos checklists são apresentar: (i) as espécies que ocorrem em uma determinada região, (ii) riqueza e diversidade do grupo em questão, (iii) ampliação da distribuição, (iv) lacunas de coletas e diminuição do viés do déficit Linneano e Wallaceano (Thomas et al. 2012; Cardoso et al. 2017; Antonelli et al. 2018).

O Brasil detém a flora mais rica do mundo (Forzza et al. 2012) e com maior número de espécies restritas (Ulloa Ulloa et al. 2017). As listas provindas até o momento sugerem alta diversidade e riqueza, principalmente para os grupos de Angiospermas (33.099 espécies), seguido de Samambaias e Licófitas (286 spp.) (BFG 2015), compreendendo as plantas vasculares. Já a flora de Mato Grosso está representada por 5.729 espécies de Angiospermas (BFG 2015). Entretanto, Forzza et al. (2012) salienta que essa região está subamostrada, com baixas densidades e lacunas de coletas (Hopkins 2007; Forzza et al. 2012; BFG 2015). Estudos e sínteses realizadas (Cardoso et al. 2017; BFG 2018) mostram Mato Grosso ainda como um Estado com baixa densidade de coletas e espécies, e várias lacunas no conhecimento das espécies que ali ocorrem, lacunas essas que podem ser preenchidas com estudos florísticos e floras locais, conforme relatado por Cardoso et al. (2017) e Ribeiro et al. (2020).

O único checklist da flora Mato-grossense mais completo até os dias de hoje foi proposto por Dubs (1998), o "Prodromus florae matogrossensis", e após 22 anos é a única referência taxonomicamente verificada da Flora do estado e ainda assim não representa nem metade da Flora já conhecida para os dias de hoje e precisa urgentemente de revisão e atualização, pois a riqueza e diversidade da Flora dos municípios está subamostrada, de acordo com observações de Almeida et al. (2015) e Ribeiro (2018).

O município de Nova Bandeirantes, localizado no norte do Estado de Mato Grosso, não apresenta nenhum tratamento florístico. Este cenário dificulta ações de conservação e até mesmo recuperação das áreas degradadas do município, uma vez que as espécies da flora nativa dessa região ainda não foram inventariadas formalmente. Ressalta-se que o município está na lista dos que mais perderam florestas nos últimas 30 anos e tem sido foco de incêndios criminosos por mais de duas décadas (MMA 2007). Frente a esse panorama, catalogar a flora dessa região se torna extremamente importante para a elaboração de políticas de uso do solo e frente ao avanço da fronteira agrícola que tem causado destruição em massa das florestas e consequentemente, há fragmentação de habitats (Laurance et al. 2000) e colapso no estoque de carbono na região sul amazônica (Bonini et al. 2018).

Embora o projeto "Flora do Brasil $2020 \mathrm{em}$ construção” (BFG 2015; 2018), tem como objetivo monografar e apresentar o maior e mais amplo tratamento taxonômico e checklist das plantas brasileiras, este estudo só revelará a riqueza em nível federal e em escala estadual, sendo que a diversidade e riqueza da flora dos municípios ainda permanece e permanecerá desconhecida. Frente a isso, estudos de checklists e inventários locais são extremamente importantes para quantificar a diversidade florística no âmbito municipal. Assim, este trabalho tem como objetivo, apresentar o (i) primeiro checklist das plantas vasculares de Angiospermas do município de Nova Bandeirantes, MT e (ii) discutir a fitogeografia, lacunas, conservação e os avanços no conhecimento da flora do Município.

\section{Material e Métodos \\ Área de estudo}

Nova Bandeirantes compreende um dos 141 municípios do Estado de Mato Grosso. Com $9.531 \mathrm{~km}^{2}$, localizado na região entre Interflúvio Juruena-Teles Pires (RADAM BRASIL 1978; IBGE 2018). São encontrados no município: florestas ombrófilas densas e abertas, submontanas e aluviais bem como formação de afloramentos rochosos, florestas estacionais, savanas florestas arborizadas (RADAM BRASIL 1978; Borges et al 2014). O município apresenta cinco tipos de solos: (i) Argissolo Vermelho-Amarelo, (ii) Gleissolo háplico, (iii) Latossolo, (iv) Neossolos Quatzarênicose e (v) Nitossolos Vermelhos (IBGE 2001). O clima é do tipo Am Köppen (Alvares et al. 2014), com pluviosidade entre $2800-3100 \mathrm{~mm}$ anuais e temperatura variando entre $24-26$ graus ${ }^{\circ} \mathrm{C}$ ao longo do ano. 


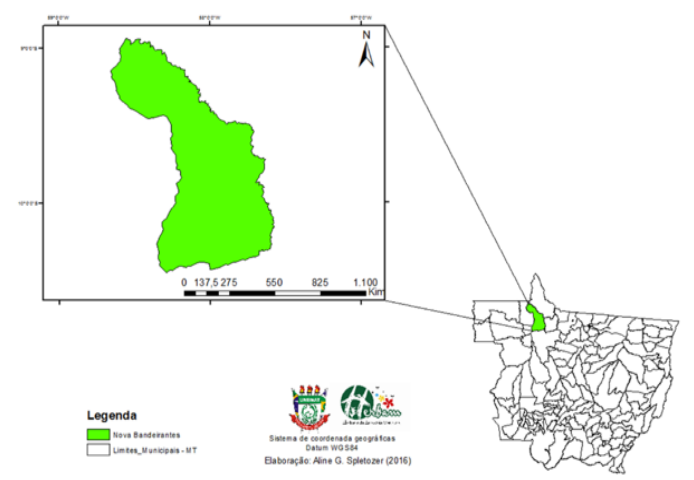

Figura 1: Mapa de localização de Nova Bandeirantes, Mato Grosso, Brasil.

\section{Inventários Florísticos}

As coletas botânicas aleatórias foram realizadas (2013-2018) seguindo metodologias de "caminhamento" descrito por Filgueiras et al. (1994). Herborizamos as coletas de acordo com Fidalgo e Bononi (1989). As exsicatas seguem depositadas no Herbário da Amazônia Meridional, da Universidade do Estado de Mato Grosso, Campus de Alta Floresta, registrado sob o acrônimo HERBAM) (Lopes 2015) (licença SISBIO 57107-2 para as coletas de R.S. Ribeiro).

\section{Construção do Banco de Dados}

Para este estudo foi considerado todo o conhecimento existente e disponibilizado via Global Biodiversity Information Facility (GBIF) e specieslink sobre a Flora Vascular de Nova Bandeirantes, MT, depositados em coleções (Herbários). Usando rotinas de linguagem de programação $\mathrm{R}$, via scripts e pacotes, extraímos todos os registros disponibilizados da flora nessas plataformas para planilhas modelos $x l s x$. No ambiente $\mathrm{R} \quad$ (https://www.r-project.org/), as planilhas foram unidas $\mathrm{e}$ as duplicatas foram retiradas via pacote Flora (https://cran.rproject.org/web/packages/flora/index.html), a nomenclatura dos táxons foi atualizada de acordo com a Flora do Brasil 2020 em construção (2019). Também foi verificado a distribuição Geográfica e Fitogeográfica dos táxons ocorrentes no município para discutir amplitude biogeográfica e aspectos fitogeográficos da flora dessa região.

\section{Apresentação do Checklist}

Os táxons foram organizados em ordem alfabética de família, gênero e epíteto. Os acrônimos dos Herbário estão de acordo com Thiers (2020) com adaptações de sub-coleções do specieslink (2019), são seguidos pelos respectivos vouchers. Os status de conservação seguem os critérios IUCN (2012) de acordo com CNCFlora (2012a). Para manter a rigorosidade taxonômica, foi considerado ao menos um voucher identificado por especialista. Considerando que nenhuma listagem florística ou tratamento taxonômico foi realizado para o município, tratamos todos os registros como inéditos para o município. Quando a espécie ainda não havia sido citada para MT, de acordo com a Flora do Brasil 2020 em construção (2019) foi indicado como potencial novo registro.

\section{Resultados e Discussão}

São reconhecidos para o município de Nova Bandeirantes, Mato Groso, Brasil, 399 registros de Flora, representam 48 famílias, 88 gêneros, 153 espécies de angiospermas. Desse total, 29 espécies são indicadas como novas ocorrências para o Estado de Mato Grosso de acordo com a Flora do Brasil em construção 2020 (2019). Dentre as 153 espécies registradas, destaque para Hymenea parvifolia Huber, considerada vulnerável, e a única espécie deste inventário enquadrada dentro dos parâmetros de risco de extinção (CNCFlora 2012b; Martinelli \& Moraes 2013). Uma espécie foi recentemente descrita e com typus oriundo do Município, Thismia ribeiroi Engels, D. Ferreira-da-Silva \& SoaresLopes, cuja coleta foi realizada durante o desenvolvimento dessa pesquisa, além de $T$. singeri Mass \& Mass, considerada nova ocorrência para o Brasil de acordo com Da Silva et al. (2020).

As famílias mais representativas para o município são: Bignoniaceae (33 spp.), Fabaceae (22 spp.), Moraceae e Rubiaceae (6 spp.), Annonaceae, Apocynaceae e Burseraceae (5 spp.), Acanthaceae, Melastomataceae e Podostemaceae (4 spp.), Asteraceae, Celastraceae, Chrysobalanaceae, Malvaceae, Meliaceae e Myrtaceae (3 spp.), seguidas de Araceae, Boraginaceae, Combretaceae, Euphorbiaceae, Lecythidaceae, Piperaceae, Urticaceae e Thismiaceae (2 spp.) as demais apresentam uma (1) espécie cada (Tabela 1).

No contexto da região sul Amazônica, as famílias mais ricas são: Fabaceae, Rubiaceae, Melastomataceae e Moraceae (Zappi et al. 2011), que corrobora para o padrão Amazônico da riqueza documentada neste trabalho. Fabaceae conta com diversos estudos realizados na região norte do Estado, pois inúmeras novidades taxonômicas e uma coleção referência da família se encontra no Herbário de acrônimo HERBAM (Lopes et al 2015; Fernandes et al. 2015; Fernandes et al. 2016a; b; Souza et al. 2016; Silva et al. 2019)

Em relação a Thismiaceae e Bignoniaceae, estas são exemplos que inventários florísticos específicos e intensos, feitos por taxonomistas especialistas regionais podem mudar o panorama e padrões de riqueza florística documentada para Amazônia. Pois, a primeira nunca havia sido registrada em listas florísticas no Mato Grosso (Da Silva et al. 2020). E a segunda, nunca apareceu em primeiro lugar em listas florísticas, cuja explicação deve-se aos extensos esforços de inventários focados especificamente para o grupo na região (Ribeiro 2018), especialidade do primeiro autor deste estudo. 
Em relação as Bignoniaceae, uma lista taxonômica compreensiva para o Mato Grosso está em andamento, baseado no compilado dos inventários realizados nos últimos anos em diferentes regiões do Estado (Ribeiro 2018; Ribeiro et al. 2018a;b;c; Ribeiro \& Soares-Lopes 2018)

Esses resultados taxonômicos ressalta principalmente, que a formação de taxonomistas focados em diferentes grupos taxonômicos, tem se mostrado promissor para diminuir os déficits taxonômicos e geográficos da flora do Norte de Mato Grosso, bem como apresentação de novidades taxonômicas para ciência, incluindo espécies novas, subespécies e híbridos, novas ocorrências e ampliações de distribuição de táxons já descritos, principalmente nos municípios circunvizinhos a Nova Bandeirantes (Koch \& Silva 2012; PetiniBenelli et al. 2014; Petini-Benelli \& Soares-Lopes 2015; Bortolan et al 2016; Fernandes et al. 2015; Fernandes et al. 2016; Ribeiro et al. 2016; Souza et al. 2016; Petini-Benelli \& Soares-Lopes 2017; Petini-Benelli \& Izzo 2017; Almeida 2018; Cardoso 2019; Ribeiro 2018; Silva et al. 2019; Fernandes et al. 2019; Koch et al. 2019; Da Silva et al 2020; Ribeiro et al. 2020; Silva et al. 2020; Ribeiro et al. 2021).

Tabela 1: Checklist das plantas vasculares (Angiospermas) no município de Nova Bandeirantes, MT.

\begin{tabular}{|c|c|c|c|}
\hline Família/ Espécie & HERBÁRIO & Voucher & CNCFlora \\
\hline \multicolumn{4}{|l|}{ Acanthaceae } \\
\hline Justicia calycina (Nees) V.A.W.Graham & ESA & 46645 & NA \\
\hline Justicia polystachya Lam. & SPF & 199761 & NA \\
\hline Ruellia costata (Nees) Hiern & SPF & 199763 & NA \\
\hline Ruellia exserta Wassh. \& J.R.I. Wood & ESA & 46729 & NA \\
\hline \multicolumn{4}{|l|}{ Amaranthaceae } \\
\hline Cyathula prostrata Blume & ESA & 48159 & NA \\
\hline \multicolumn{4}{|l|}{ Anacardiaceae } \\
\hline Astronium lecointei Ducke & UFMT & 28001 & NA \\
\hline \multicolumn{4}{|l|}{ Annonnaceae } \\
\hline Duguetia lanceolata A.St.-Hil.* & UFMT & 28011 & $\mathrm{LC}$ \\
\hline Oxandra xylopioides Diels & UFMT & 28012 & NA \\
\hline Unonopsis guatterioides (A.DC.) R.E.Fr. & ESA & 48176 & NA \\
\hline Xylopia langsdorffia A.St.-Hil. \& Tul. * & ESA & 48177 & NA \\
\hline Xylopia polyantha R.E.Fr. & ESA & 48200 & NA \\
\hline \multicolumn{4}{|l|}{ Apocynaceae } \\
\hline Couma macrocarpa Barb.Rodr. & UFMT & 28677 & NA \\
\hline Hemipogon acerosus Decne. & ESA & 48213 & NA \\
\hline Himatanthus articulatus (Vahl) Woodson & ESA & 49334 & NA \\
\hline Himatanthus sucuuba Wood. * & MBM & 272031 & NA \\
\hline Mandevilla funiformis (Vell.) K.Schum. * & HUEFS & 70124 & NA \\
\hline \multicolumn{4}{|l|}{ Araceae } \\
\hline Heteropsis oblongifolia Kunth & ESA & 49350 & NA \\
\hline Heteropsis spruceana Schott * & ESA & 49349 & NT \\
\hline \multicolumn{4}{|l|}{ Araliaceae } \\
\hline Schefflera morototoni (Aubl.) Maguire et al. & SPF & 142668 & NA \\
\hline \multicolumn{4}{|l|}{ Asteraceae } \\
\hline Mikania divaricata Poepp. \& Endl. & ESA & 46511 & NA \\
\hline Mikania lindleya DC. & SP & 357721 & $\mathrm{LC}$ \\
\hline
\end{tabular}


...continuação

\begin{tabular}{|c|c|c|c|}
\hline Mikania parviflora (Aubl.) H.Karst. & UEC & 111515 & NA \\
\hline \multicolumn{4}{|l|}{ Bignoniaceae } \\
\hline Adenocalymma biternatum (A.Samp.) L.G.Lohmann & ESA & 46520 & NA \\
\hline Adenocalymma calcareum Udulutsch \& P. Dias & UEC & 114334 & NA \\
\hline Adenocalymma impressum (Rusby) Sandwith & UFMT & 30095 & NA \\
\hline Adenocalymma subincanum Huber & ESA & 48025 & NA \\
\hline Amphilophium paniculatum (L.) Kunth & ESA & 48024 & NA \\
\hline Anemopaegma paraense Bureau \& K.Schum. * & FLOR & 66624 & NA \\
\hline Anemopaegma setilobum A.H.Gentry * & UFMT & 34702 & NA \\
\hline Bignonia noterophila Mart. ex DC. & UFMT & 34701 & NA \\
\hline Callichlamys latifolia (Rich.) K.Schum. & UEC & 113731 & NA \\
\hline Cuspidaria floribunda (DC.) A.H.Gentry & UEC & 113730 & NA \\
\hline Cuspidaria sceptrum (Cham.) L.G.Lohmann & SPF & 222220 & NA \\
\hline Dolichandra quadrivalvis (Jacq.) L.G.Lohmann & SPF & 222219 & NA \\
\hline Dolichandra unguis-cati (L.) L.G.Lohmann & SPF & 222218 & NA \\
\hline Fridericia dichotoma (Jacq.) L.G.Lohmann & SPF & 222217 & NA \\
\hline Fridericia florida (DC.) L.G.Lohmann & SPF & 222216 & NA \\
\hline Fridericia ornithophila (A.H.Gentry) L.G.Lohmann & SPF & 222230 & NA \\
\hline Fridericia platyphylla (Cham.) L.G.Lohmann & SPF & 222223 & NA \\
\hline Fridericia speciosa Mart. * & SPF & 222256 & NA \\
\hline Fridericia triplinervia (Mart. ex DC.) L.G.Lohmann & SPF & 222254 & NA \\
\hline Handroanthus ochraceus (Cham.) Mattos & SPF & 221821 & NA \\
\hline Handroanthus serratifolius (Vahl) S.Grose & SPF & 222227 & NA \\
\hline Lundia corymbifera (Vahl) Sandwith $*$ & SPF & 221827 & NA \\
\hline Manosella cordifolia (DC.) A.H.Gentry & SPF & 221819 & NA \\
\hline Pachyptera kerere (Aubl.) Sandwith * & SPF & 222214 & NA \\
\hline Pyrostegia venusta (Ker Gawl.) Miers & SPF & 222213 & NA \\
\hline Sparattosperma leucanthum (Vell.) K.Schum. & SPF & 221825 & NA \\
\hline Stizophyllum riparium (Kunth) Sandwith & UFMT & 30765 & NA \\
\hline Tabebuia aurea (Silva Manso) Benth. \& Hook.f. ex S.Moore & UFMT & 30769 & NA \\
\hline Tanaecium pyramidatum (Rich.) L.G.Lohmann & SPF & 221808 & NA \\
\hline Tynanthus panurensis (Bureau) Sandwith * & SPF & 221809 & NA \\
\hline Tynanthus polyanthus (Bureau) Sandwith & ESA & 41991 & NA \\
\hline Xylophragma platyphyllum (DC.) L.G.Lohmann & ESA & 41947 & NA \\
\hline Xylophragma myrianthum (Cham.) Sprague & ESA & 41941 & NA \\
\hline \multicolumn{4}{|l|}{ Bixaceae } \\
\hline Bixa arborea Huber & SPF & 222247 & $\mathrm{LC}$ \\
\hline \multicolumn{4}{|l|}{ Boraginaceae } \\
\hline Cordia exaltata Lam. & SPF & 222248 & NA \\
\hline Cordia nodosa Lam. & SPF & 222249 & NA \\
\hline \multicolumn{4}{|l|}{ Burseraceae } \\
\hline Protium carnosum A.C.Sm.* & SPF & 222253 & NA \\
\hline
\end{tabular}


...continuação

\begin{tabular}{|c|c|c|c|}
\hline Protium decorum Daly & ESA & 44217 & NA \\
\hline Protium robustum (Swart) D.M.Porter & SPF & 222215 & NA \\
\hline Protium sagotianum Marchand & SPF & 222211 & NA \\
\hline Protium spruceanum (Benth.) Engl. & UFMT & 31094 & NA \\
\hline \multicolumn{4}{|l|}{ Celastraceae } \\
\hline Cheiloclinium belizense (Standl.) A.C. Sm. & ESA & 42020 & NA \\
\hline Cheiloclinium cognatum (Miers) A.C.Sm. & SPF & 222212 & NA \\
\hline Salacia impressifolia (Miers) A.C. Sm. & SPF & 222283 & NA \\
\hline \multicolumn{4}{|l|}{ Chrysobalanaceae } \\
\hline Hirtella gracilipes (Hook.f.) Prance & SPF & 222268 & NA \\
\hline Hirtella racemosa Lam. & HERBAM & 11621 & $\mathrm{LC}$ \\
\hline Hirtella sprucei Benth. ex Hook.f. & ESA & 42058 & NA \\
\hline \multicolumn{4}{|l|}{ Clusiaceae } \\
\hline Symphonia globulifera L.f. & SPF & 222257 & NA \\
\hline \multicolumn{4}{|l|}{ Combretaceae } \\
\hline Combretum laxum Jacq. & SPF & 222207 & NA \\
\hline Combretum mellifluum Eichler & SPF & 221830 & NA \\
\hline \multicolumn{4}{|l|}{ Connaraceae } \\
\hline Connarus suberosus Planch. & SPF & 222231 & NA \\
\hline \multicolumn{4}{|l|}{ Cucurbitaceae } \\
\hline Gurania bignoniacea (Poepp. \& Endl.) C.Jeffrey & SPF & 222210 & NA \\
\hline \multicolumn{4}{|l|}{ Ebenaceae } \\
\hline Diospyros longifolia (Spruce ex Engl.) Sleumer \& F.White * & SPF & 222208 & NA \\
\hline \multicolumn{4}{|l|}{ Elaeocarpaceae } \\
\hline Sloanea obtusa (Splitg.) Schum. & SPF & 222206 & NA \\
\hline \multicolumn{4}{|l|}{ Euphorbiaceae } \\
\hline Manihot baccata Allem & UFMT & 31945 & NA \\
\hline Manihot esculenta Crantz & ESA & 48738 & NA \\
\hline \multicolumn{4}{|l|}{ Fabaceae/Leguminosae } \\
\hline Aeschynomene america $\mathrm{L}$. & UFMT & 29233 & NA \\
\hline Albizia pedicellaris (DC.) L.Rico & ESA & 42135 & NA \\
\hline Andira cuyabensis Benth. & UFMT & 29218 & NA \\
\hline Bauhinia forficata Link * & ESA & 42147 & NA \\
\hline Bauhinia rufa (Bong.) Steud. * & UEC & 112769 & NA \\
\hline Canavalia grandiflora Benth. & NYBG_BR & 1416205 & NA \\
\hline Centrosema bracteosum Benth. & NYBG_BR & 1416206 & $\mathrm{LC}$ \\
\hline Dioclea virgata (Rich.) Amshoff & NYBG_BR & 1416207 & NA \\
\hline Enterolobium maximum Ducke & ESA & 42232 & NA \\
\hline Hymenea parvifolia Huber & NYBG_BR & 929620 & VU \\
\hline Inga alba (Sw.) Willd. & ESA & 42208 & NA \\
\hline Leptolobium dasycarpum Vogel & UFMT & 29286 & NA \\
\hline Leptolobium elegans Vogel & UEC & 112047 & NA \\
\hline
\end{tabular}


...continuação

\begin{tabular}{|c|c|c|c|}
\hline Macrolobium gracile Spruce ex Benth. & HRCB & 74980 & NA \\
\hline Schnella smilacina (Spreng.) G.Don * & ESA & 38543 & NA \\
\hline Senegalia altiscandens (Ducke) Seigler \& Ebinger * & HUEFS & 34688 & NA \\
\hline Senegalia giganticarpa (G.P.Lewis) Seigler \& Ebinger * & ESA & 38564 & NA \\
\hline Senegalia polyphylla (DC.) Britton \& Rose & MBM & 235340 & NA \\
\hline Senna gardneri (Benth.) H.S.Irwin \& Barneby * & ESA & 42881 & NA \\
\hline Senna multijuga (Rich.) H.S.Irwin \& Barneby & ESA & 42895 & NA \\
\hline Senna tapajozensis (Ducke) H.S.Irwin \& Barneby & ESA & 42896 & NA \\
\hline Zygia inundata (Ducke) H.C.Lima ex Barneby \& Grimes * & ESA & 42902 & NA \\
\hline \multicolumn{4}{|l|}{ Hypericaceae } \\
\hline Vismia bemerguii M.E.Berg & ESA & 42901 & NA \\
\hline \multicolumn{4}{|l|}{ Lamiaceae } \\
\hline Aegiphila bracteolosa Moldenke & MBM & 223784 & NA \\
\hline \multicolumn{4}{|l|}{ Lauraceae } \\
\hline Nectandra leucantha Nees * & ESA & 42994 & NA \\
\hline \multicolumn{4}{|l|}{ Lecythidaceae } \\
\hline Cariniana domestica (Mart.) Miers & UEC & 94190 & NA \\
\hline Eschweilera parvifolia Mart. ex DC. & ESA & 43058 & NA \\
\hline \multicolumn{4}{|l|}{ Loganiaceae } \\
\hline Spigelia anthelmia L. & ESA & 43064 & NA \\
\hline \multicolumn{4}{|l|}{ Malvaceae } \\
\hline Apeiba echinata Gaertn. & ESA & 43097 & NA \\
\hline Eriotheca pubescens (Mart. \& Zucc.) Schott \& Endl. & UFMT & 30334 & $\mathrm{LC}$ \\
\hline Theobroma subincanum Mart. & UFMT & 30314 & NA \\
\hline \multicolumn{4}{|l|}{ Melastomataceae } \\
\hline Aciotis indecora (Bonpl.) Tria & ESA & 43192 & NA \\
\hline Miconia nervosa (Sm.) Tria & UFMT & 28409 & NA \\
\hline Miconia valtheri udin * & ESA & 40849 & NA \\
\hline Mouriri sagotiana Tria & SJRP & 33491 & NA \\
\hline \multicolumn{4}{|l|}{ Meliaceae } \\
\hline Guarea kunthiana A.Juss. & ESA & 37969 & NA \\
\hline Guarea macrophylla Vahl & HUEFS & 164181 & NA \\
\hline Trichilia micrantha Benth. & ESA & 38107 & NA \\
\hline \multicolumn{4}{|l|}{ Menispermaceae } \\
\hline Abuta sandwithiana Krukoff \& Barneby & HUEFS & 164170 & NA \\
\hline \multicolumn{4}{|l|}{ Moraceae } \\
\hline Brosimum rubescens Taub. & SORO & 6057 & NA \\
\hline Helicostylis tomentosa (Poepp. \& Endl.) Rusby & ESA & 40951 & LC \\
\hline Perebea guianensis Aubl. * & ESA & 44200 & NA \\
\hline Pseudolmedia laevigata Trécul & ESA & 38178 & NA \\
\hline Pseudolmedia laevis (Ruiz \& Pav.) J.F.Macbr. & UEC & 114274 & NA \\
\hline Pseudolmedia macrophylla Trécul & ESA & 42417 & NA \\
\hline
\end{tabular}


...continuação

\begin{tabular}{|c|c|c|c|}
\hline \multicolumn{4}{|l|}{ Myristicaceae } \\
\hline Iryanthera juruensis Warb. & ESA & 38439 & NA \\
\hline \multicolumn{4}{|l|}{ Myrtaceae } \\
\hline Eugenia egensis DC. & CGMS & 33000 & NA \\
\hline Eugenia florida DC. & ESA & 38251 & $\mathrm{LC}$ \\
\hline Eugenia hiemalis Cambess. * & UFMT & 33444 & $\mathrm{LC}$ \\
\hline \multicolumn{4}{|l|}{ Nyctaginaceae } \\
\hline Neea hermaphrodita S.Moore & ESA & 42605 & NA \\
\hline \multicolumn{4}{|l|}{ Orchidaceae } \\
\hline Xylobium foveatum (Lindl.) Nichols & VIC & 18342 & NA \\
\hline \multicolumn{4}{|l|}{ Passifloraceae } \\
\hline Passiflora coccinea Aubl. & VIC & 18344 & NA \\
\hline \multicolumn{4}{|l|}{ Peraceae } \\
\hline Pera eiteniorum Bigio \& Secco & ESA & 39538 & NA \\
\hline \multicolumn{4}{|l|}{ Piperaceae } \\
\hline Piper hispidum Sw. & ESA & 38167 & NA \\
\hline Piper pubisubmargilum Yunck. * & ESA & 38173 & $\mathrm{LC}$ \\
\hline \multicolumn{4}{|l|}{ Podostemaceae } \\
\hline Castelnavia fluitans Tul. \& Wedd. & ESA & 42622 & NA \\
\hline Castelnavia princeps Tul. \& Wedd. & MBM & 287526 & NA \\
\hline Tristicha trifaria (Bory ex Willd.) Spreng. & UEC & 114467 & NA \\
\hline Weddellina squamulosa Tul. & ESA & 45264 & NA \\
\hline \multicolumn{4}{|l|}{ Primulaceae } \\
\hline Clavija nutans (Vell.) B.Ståhl & VIC & 46437 & NA \\
\hline \multicolumn{4}{|l|}{ Rhamnaceae } \\
\hline Colubrina glandulosa Perkins & ESA & 44133 & $\mathrm{LC}$ \\
\hline \multicolumn{4}{|l|}{ Rubiaceae } \\
\hline Capirona decorticans Spruce & ESA & 44132 & NA \\
\hline Isertia hypoleuca Benth. & UEC & 114468 & NA \\
\hline Palicourea racemosa (Aubl.) Borhidi & ESA & 42761 & $\mathrm{LC}$ \\
\hline Psychotria colorata (Willd. ex Schult.) Müll.Arg. * & ESA & 45216 & NA \\
\hline Psychotria deflexa DC. & VIC & 18343 & NA \\
\hline Uncaria guianensis (Aubl.) J.F.Gmel. & UEC & 94117 & NA \\
\hline Ticorea longiflora DC. * & HUEFS & 33671 & NA \\
\hline \multicolumn{4}{|l|}{ Salicaceae } \\
\hline Casearia sylvestris $\mathrm{Sw}$. & ESA & 38835 & NA \\
\hline \multicolumn{4}{|l|}{ Solanaceae } \\
\hline Solanum placitum C.V.Morton & UFMT & 28329 & NA \\
\hline \multicolumn{4}{|l|}{ Thismiaceae } \\
\hline Thismia melanomitra Mass \& Mass * & HERBAM & & NA \\
\hline Thismia ribeiroi Engels, D. Ferreira-da-Silva \& Soares-Lopes * & HERBAM & & NA \\
\hline Urticaceae & & & \\
\hline
\end{tabular}


...continuação

\begin{tabular}{llcc}
\hline Cecropia concolor Willd. & ESA & 46379 & NA \\
$\begin{array}{l}\text { Cecropia sciadophylla Mart. } \\
\text { Violaceae }\end{array}$ & ESA & 42110 & NA \\
Rinoreocarpus ulei (Melch.) Ducke & ESA & 47605 & NA
\end{tabular}

Em astericos (*) novos registros para MT de acordo com Flora do Brasil em construção (2019). Status de ameaça de acordo com CNCFlora (2012) (NA: Não Avaliado, LC: Pouco preocupante, NT: Quase ameaçada e VU: Vulnerável)

Foi identificado os seguintes padrões de hábitos: Árvore (55 spp.), Liana (42 spp.), ArbustoÁrvore (20 spp.), Arbusto (9 spp.), Erva (7 spp.), Arbusto-Árvore-Liana (4 spp.), Arbusto-Liana (3 spp.), Erva-Subarbustos (3 spp.), Arbusto-ÁrvoreSubarbusto (2 spp.), Subarbusto (2 spp.) e ArbustoSubarbusto (1 spp.). a elevada riqueza de árvore aqui, pode ser explicado pelo efeito coletor e por haver uma maior tendência em inventário de árvores nessa região (Cardoso et al. 2017; ter Steege et al. 2019).

A Flora do Município de Nova Bandeirantes apresentou 20 padrões fitogeográficos: Amazônia (43 espécies), Amazônia-Cerrado-Mata Atlântica (22 spp.), Amazônia-Cerrado (19 spp.), AmazôniaMata Atlântica (10 spp.), Amazônia-CaatingaCerrado-Mata Atlântica (9 spp.), AmazôniaCaatinga-Cerrado-Mata Atlântica-Pantanal (9 spp.), Mata Atlântica (8 spp.), Amazônia-Cerrado-Mata Atlântica-Pantanal (6 spp.), Cerrado (5 spp.), Amazônia-Caatinga-Cerrado-Mata AtlânticaPampa-Pantanal (4 spp.), Cerrado-Mata Atlântica (3 spp.), Caatinga-Cerrado-Mata Atlântica (2 spp.), Amazônia-Caatinga (1 spp.), Amazônia-CaatingaCerrado (1 spp.), Amazônia-Caatinga-Cerrado-Mata Atlântica-Pampa (1 spp.), Amazônia-Caatinga-Mata Atlântica-Pantanal (1 spp.), Amazônia-CerradoPantanal (1 spp.), Amazônia-Mata AtlânticaPantanal (1 spp.), Amazônia-Pantanal (1 spp.) e Caatinga (1 spp.) (Flora do Brasil em construção 2019). Uma abordagem similar foi realizada por Ribeiro et al. (2016), onde este encontrou padrões similares para Bignoniaceae e Gallo et al. (2017) para Zingiberales no sul da Amazônia.
Das espécies ocorrentes nesse município, 68\% (105 spp.) tem padrão Amazônico e aproximadamente $42 \%$ são restritos a esse Bioma. Atualmente, existem algumas contradições na fitogeografia desta região, evidenciado pela alta diversidade de fitofisionomias (RADAM BRASIL 1978; Zappi et al. 2011; Soares-Lopes et al. 2016a;b), incluindo a tradicional proposta de região de transição (Ab'Saber 1977). Este estudo inferiu que Flora do município de Nova Bandeirantes é taxonomicamente mais similar ao Bioma Amazônico, corroborando com as novas propostas de bioregionalização da paisagem dos limites do Bioma Amazônico mato-grossense (Marques et al. 2019).

As comparações realizadas com as listas de flora de municípios circunvizinhos e localidades ao longo da Amazônia Mato-grossense (Tabela 2) evidenciam as altas lacunas de coletas na borda sul amazônica já documentadas em Hopkins (2007) e a sub-amostragem florística e taxonômica discutida por Forzza et al. (2010; 2012). Estudos florísticos em regiões tropicais salientam que para considerar uma área geográfica bem amostrada taxonomicamente, são necessárias três amostras ou registros $/ \mathrm{km}^{2}$, no mínimo (Shepherd 2003; Cielo-Filho et al. 2009). Em Nova Bandeirantes há apenas 0,2 amostras por $\mathrm{km}^{2}$. Esses dados indicaram que o conhecimento florístico dessa região está abaixo do esperado, uma tendência pela região sul amazônica e para o Estado de Mato Grosso (Almeida 2018; Ribeiro \& SoaresLopes 2018; Cardoso 2019) (Tabela 2).

Tabela 2: Estudos florísticos municipais e regionais na Amazônia Mato-grossense e densidade de registros por $\mathrm{km}^{2}$ e riqueza de espécie.

\begin{tabular}{lcccc}
\multicolumn{1}{c}{ Locais } & registros & $\begin{array}{c}\text { n. } \\
\text { spp. }\end{array}$ & Área & registos $/ \mathbf{k m}^{2}$ \\
\hline Aripuanã, MT (Almeida 2018) & 4296 & 1042 & $25.049,00 \mathrm{~km}^{2}$ & $0,00017 / \mathrm{km}^{2}$. \\
\hline Nova Bandeirantes, MT (este estudo) & 399 & 153 & $9.531,00 \mathrm{~km}^{2}$ & $0,2 / \mathrm{km}^{2}$ \\
\hline Colíder, MT (Cardoso 2019) & 3654 & 765 & $3.093,173 \mathrm{Km}^{2}$ & $1,20 / \mathrm{km}^{2}$ \\
\hline $\begin{array}{l}\text { Região do Cristalino (Zappi et al. } \\
\text { 2011) }\end{array}$ & 3500 & 1301 & $1.347,600 \mathrm{~km}^{2}$ & $3,85 / \mathrm{KM}^{2}$
\end{tabular}




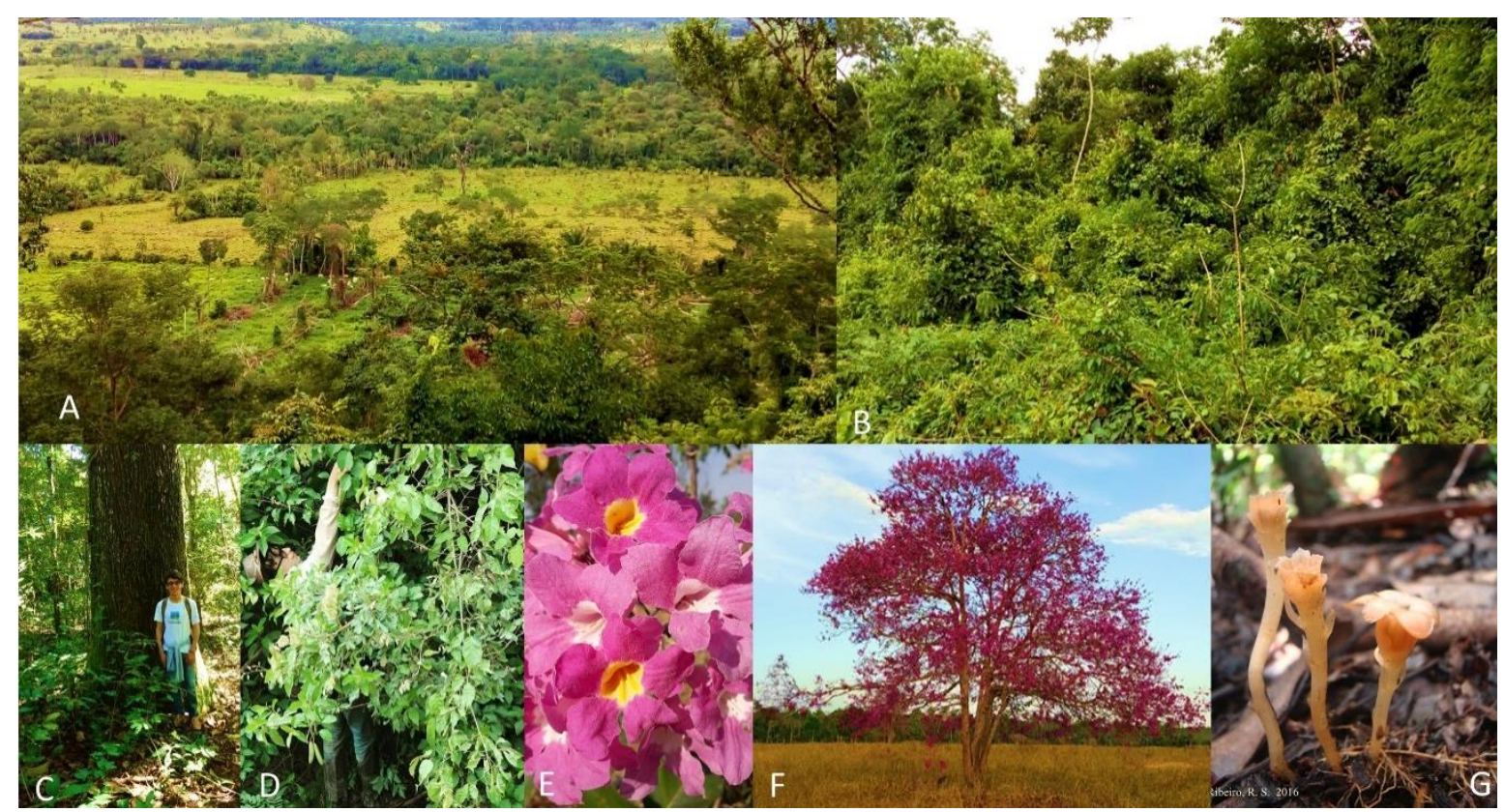

Figura 2. A- Fragmentação florestal. B- Floresta ombrófila aberta. C e D- Expedições de campo. E- Bignoniaceae: Xylophragma myrianthum (Cham.) Sprague. F - Erythroxylaceae. G - Thismiaceae: Thismia ribeiroi Engels, D. Ferreira-da-Silva \& Soares-Lopes sp. nov. in Phytotaxa 429 (4): 261-273 (Da Silva et al. 2020).

\section{Conclusões}

O Município de Nova Bandeirantes possui 153 espécies e 48 famílias de Angiospermas. Destas, 29 espécies foram indicadas como novos registros para Mato Grosso. Os padrões fitogeográficos da flora inferiram que há mais espécies Amazônicas do que de área secas (Cerrado e Caatinga). Também foi evidenciado que a flora dessa região compartilha espécies com a Mata Atlântica, incluindo espécies disjuntas. Essa grande diversidade de padrões faz desse munícipio uma região importante para se entender a origem estrutural da biota Sul Amazônica. Para isso, são necessários inventários taxonômicos urgentes no Município, visto que a região possui baixa densidade de coletas botânicas.

\section{Agradecimentos}

Os autores agradecem ao Herbário da Amazônia Meridional e Universidade do Estado de Mato Grosso, Campus Alta Floresta pela infraestrutura e logística. RSR e CRASL, Bolsa CNPq PIBIC do primeiro autor (RSR PIBIC/UNEMAT 126114/2016-1), (800158/2016- 4). RSR, bolsa PCIDD no Instituto Nacional da Mata Atlântica -INMA (2019-atual) (Processo: 301395/2021-7 INMA/CNPq/MCTI). Os autores agradecem a Adriani M. S. Ribeiro por providenciar fotos da Flora de Nova Bandeirantes, MT e Aline Spletozer pela confecção do mapa.

\section{Referências}

Ab'Saber AN (1977) Os domínios morfoclimáticos na América do Sul: primeira aproximação. Geomorfologia, São Paulo 52: 1-22.
Almeida AASD, Ribeiro RS, Spletozer AG, Rodrigues L, Silva DR, Teixeira FC, Lopes CRAS (2016) Checklist e distribuição geográfica de Ficus L. (Moraceae Gaudich.) na Amazônia Norte MatoGrossense. Scientific Electronic Archives, 9: 9-11.

Almeida AASDD, Lopes CRAS, Ribeiro RDS, Lopes FJA, Cabral FF (2015) Bignoniaceae Juss. (Lamiales) da região da Pousada Mantega, Amazônia Meridional, Mato Grosso: distribuição e uso. In: $\sigma^{a}$ Jornada Científica da Unemat, Cáceres, Mato Grosso.

Almeida AASD (2018) Dinâmica de fragmentação florestal e checklist de Angiospermas do Município de Aripuanã, Mato Grosso, Brasil. Trabalho de Conclusão de Curso, Universidade do Estado de Mato Grosso, 64p.

Alvares CA, Stape JL, Sentelhas PC, Gonçalves JLM, Sparovek G (2013) Köppen`s climate classification map for Brazil. Meteorologische Zeitschrift, 22 (6): 711-728.

Antonelli A, Ariza M, Albert J, Andermann T, Azevedo J, Bacon C, Faurby S, Guedes T, Hoorn C, Lohmann LG, Matos-Maravi P, Ritter CD, Sanmartin I, Silvestro D, Tejedor M, Ter Steege H, Tuomisto H, Werneck FP, Zizka A, Edwards SV (2018). Conceptual and empirical advances in Neotropical biodiversity research. PeerJ. 6: e5644. doi: $10.7717 /$ peerj. 5644 
BFG (2015) Growing knowledge: an overview of Seed Plant diversity in Brazil. Rodriguésia, 66: 1085-1113.

BFG (2018) Innovation and collaboration to meet Target 1 of the Global Strategy for Plant Conservation (GSPC). Rodriguésia, 69(4): 15131527.

Bicudo CEM (2004) Taxonomia. Biota Neotropica, 4(1): 1-2. doi: 10.1590/S1676-06032004000100001

Bonini I, Marimon-Junior BH, Matricardi E, Phillips OL, Petter F, Schwantes-Marimon B (2018) Collapse of ecosystem carbon stocks due to forest conversion to soybean plantations at the AmazonCerrado transition. Forest Ecology and Management, 414: 64-73. doi: 10.1016/j.foreco.2018.01.038

Borges HB, Silveira EA, Vendramin LN (2014) Flora Arbórea de Mato Grosso: tipologias vegetais e suas espécies. Entrelinhas. Cuiabá. 259p.

Bortolan GB; Koch AK, Petini-Benelli A, Rodrigues L, Saores-Lopes CRAS (2016) Gênero Catasetum Rich. ex Kunth (Orchidaceae) na área de influência da UHE Teles Pires, Mato Grosso, Brasil. Scientific Electronic Archives, 9: 87-89.

Campbell DG (1989) The importance of floristic inventory in the tropics. In: Campbell DG, Hammond HD (ed.). Floristic inventory of tropical countries. The New York Botanical Garden, New York. 5-30p.

Cardoso BF (2019) Checklist e os Padrões Fitogeográficos da Flora Vascular do município de Colíder, Mato Grosso, Brasil. Trabalho de Conclusão de Curso. Universidade do Estado de Mato Grosso. 84p.

Cardoso D, Särkinen T, Alexander S, Amorim AM, Bittrich V, Celis M, Daly DC, Fiaschi P, Funk VA, Giacomin LL, Goldenberg R, Heiden G, Iganci J, Kelloff CL, Knapp S, Lima HC, Machado AFP, Santos RM, Mello-Silva R, Michelangeli FA, Mitchell J, Moonlight P, Moraes PRL, Mori SA, Nunes TS, Pennington TD, Pirani JR, Prance GT, Queiroz LP, Rapini A, Riina R, Rincon CAV, Roque N, Shimizu G, Sobral M, Stehmann JR, Stevens WD, Taylor CM, Trovó M, van den Berg C, van der Werff H, Viana PL, Zartman CE, Forzza RC (2017) Amazon plant diversity revealed by a taxonomically verified species list. PNAS, 114: 10695-10700.

Cielo-Filho R, Baitello JB, Pastore JA, Aguiar OT, Souza SCPM, Toniato MTZ, Lima CR, Ribeiro AP (2009) Ampliando a densidade de coletas botânicas na região da bacia hidrográfica do Alto Paranapanema: Caracterização florística da Floresta Estadual e da Estação Ecológica de Paranapanema. Biota Neotropica 9: 255-276.

CNCFlora (2012a). Lista Vermelha da flora brasileira versão 2012.2 Centro Nacional de Conservação da Flora. Disponível em: $<$ http://cncflora.jbrj.gov.br/portal/ptbr/listavermelha> Acesso 14 de Julho de 2020.

CNCFlora (2012b) Hymenaea parvifolia in Lista Vermelha da flora brasileira versão 2012.2 Centro Nacional de Conservação da Flora. Disponível em <http://cncflora.jbrj.gov.br/portal/pt-

br/profile/Hymenaea parvifolia>. Acesso em 4 janeiro 2021.

Da Silva DF, Engels ME, Soares-Lopes CRA (2020) Novelties in Thismia (Thismiaceae) from South Brazilian Amazon with the description of a new species. Phytotaxa, 429(4): 261-273. doi: 10.11646/phytotaxa.429.4.2

Da Silva JV, Lopes CRAS, Fernandes JM (2016) Contribuição taxonômica ao estudo do gênero Bauhinia L. (Leguminosae) no Estado de Mato Grosso. Enciclopédia Biosfera,13: 306-314.

Dubs B (1998) Prodromus Florae Matogrossensis: Part I. Checklist of Angiosperms; Part II. Types from Mato Grosso, Partes 1-2, Betrona-Verlag, Küsnacht. 444p.

Dutra VF, Alves-Araújo A, Carrijo TT (2015) Angiosperm Checklist of Espírito Santo: using electronic tools to improve the knowledge of an Atlantic Forest biodiversity hotspot. Rodriguésia, 66(4): 1145-1152. doi: 10.1590/21757860201566414

Fernandes JM, Soares-Lopes CRA, Ribeiro RS, Silva DR (2015) Leguminosae no Acervo do herbário da Amazônia Meridional, Alta floresta, Mato Grosso. Enciclopédia Biosfera, 11(21): 22722293.

Fernandes JM, Da Costa RD, Soares-Lopes CRA, (2016a) Taxonomia de Inga macrophylla Humb. \& Bonpl. ex Willd. (Leguminosae, Mimosoideae): Uma nova ocorrência para Mato Grosso, Brasil. Enciclopédia Biosfera, 13: 1329-1335. doi: 10.18677/EnciBio_2016B_123

Fernandes JM, Rodrigues L, Piva JH, Lopes CRAS (2016b) Contribuição taxonômica ao estudo do gênero Calliandra Benth. (Leguminosae) no Estado de Mato Grosso, Brasil, Enciclopédia Biosfera, 13: 315-321. 
Fernandes JM, Soares-Lopes CRA, Fagundes O, Santos P, Almeida, AASD (2019) Zapoteca scutellifera (Leguminosae): Uma nova ocorrência em Mato Grosso, com chave de identificação para as espécies do gênero no Brasil. Enciclopédia Biosfera, 16:30, 648-661. doi: 10.18677/EnciBio_2019B60

Fidalgo O, Bononi VLR (1989) Técnicas de coleta, preservação e herborização do material botânico. São Paulo: Instituto de Botânica, 61p.

Filgueiras TS, Brochado AL, Nogueira PE, Gualla Ii GF (1994) Caminhamento - Um método expedito para levantamentos florísticos qualitativos. In: Caderno de Geociência IBGE. 12: 39-43.

Flora do Brasil 2020 em construção (2019) Jardim Botânico do Rio de Janeiro. Disponível em: <http://floradobrasil.jbrj.gov.br/ >. Acesso em: 30 nov. 2019

Forzza RC, Baumgratz JFA, Bicudo CEM, Carvalho Jr. AA, Costa A, Costa DP, Hopkins M, Leitman PM, Lohmann LG, Maia LC, Martinelli G, Menezes M, Morim MP, Coelho MAN, Peixoto AL, Pirani JR, Prado J, Queiroz LP, Souza VC, Stehmann JR, Sylvestre LS, Walter BMT, Zappi D (2010) Catálogo de plantas e fungos do Brasil. Vol. 1; Vol. 2. Andrea Jakobsson Estúdio Editorial, Jardim Botânico do Rio de Janeiro, Rio de Janeiro. 870p.; 830p.

Forzza RC, Baumgratz JFA, Bicudo CEM, Canhos DAL, Carvalho Jr. AA, Costa A, Costa DP, Hopkins M, Leitman PM, Lohmann LG, NicLughadha E, Maia LC, Martinelli G, Menezes M, Morim MP, Coelho MAN, Peixoto AL, Pirani JR, Prado J, Queiroz LP, Souza S, Souza VC, Stehmann JR, Sylvestre LS, Walter BMT, Zappi D (2012) New Brazilian floristic list highlights conservation challenges. BioScience 62: 39-45. doi: 10.1525/bio.2012.62.1.8

Gallo SC, Silva DR, Ribeiro RS, Carnicer C, Eisenlohr PV (2017). Padrões de distribuição biogeográfica das Zingiberales Grisebach em floresta estacional da Amazônia mato-grossense, Brasil. In. XIII Congresso de Ecologia, III International Symposium of Ecology and Evolution. Viçosa.

Hopkins MJ (2007) Modelling the known and unknown plant biodiversity of the Amazon Basin. Journal of Biogeography, 34(8): 1400-1411. doi: 10.1111/j.1365-2699.2007.01737.x

IBGE - Instituto Brasileiro de Geografia e Estatística (2001) Mapas de solos do brasil 2001. Disponível em: <https://mapas.ibge.gov.br/tematicos/solos> Acesso 16 julho 2020.
IBGE - Instituto Brasileiro de Geografia e Estatística (2018) Cidades e Estados. Disponível em: <https://mapas.ibge.gov.br/tematicos/solos > Acesso 16 julho 2020.

IUCN (2012) Red List categories and criteria, version 3.1, second edition. Gland and Cambridge: IUCN. 4: 32p.

Koch AK, Silva CA (2012) Orquídeas Nativas de Mato Grosso. 1. ed. Cuiabá: Carlini \& Caniato Editoração, 112p.

Koch AK, Engels ME, Veras NN, Soares-Lopes CRA (2019) A new subspecies and taxonomic notes on Passiflora L. (Passifloraceae) in Brazilian Amazon, Mato Grosso, Brazil. Phytotaxa, 402: 1320.

Lanna J, da Silva L, Morim M, Leitman P, Queiroz N, Filardi F, Dalcin E, Oliveira F, Forzza R C (2018) Herbarium collection of the Rio de Janeiro Botanical Garden (RB), Brazil. Biodiversity Data Journal, 6: e22757. doi: 10.3897/bdj.6.e22757

Laurance W, Vasconcelos H, Lovejoy T (2000) Forest loss and fragmentation in the Amazon: Implications for wildlife conservation. Oryx, 34(1): 39-45. doi: 10.1046/j.1365-3008.2000.00094.x

Lopes CRAS, Ribeiro RS, Rodrigues L, Cabral FF, Silva DR (2014) Check list de Angiospermas da Região de Influência da UHE Sinop, Médio Teles Pires, Mato Grosso. Enciclopédia Biosfera, 10: 2036-2048.

Lopes CRAS (2015) Herbário da Amazônia Meridional, Mato Grosso (HERBAM). UNISANTA Bioscience, 4 (6): 36-39.

Marques EQ, Marimon-Junior BH, Marimon BS, Matricardi EA, Mews HA, Colli GR (2019) Redefining the Cerrado- Amazonia transition: implications for conservation. Biodiversity Conservation, 1-17. doi: 10.1007/s10531-01901720-z

Martinelli G, Moraes MA (2013) Livro vermelho da flora do Brasil. Andrea Jakobsson Estúdio Editorial, Jardim Botânico do Rio de Janeiro, Rio de Janeiro. $1100 \mathrm{p}$

MMA - Ministério do Meio Ambiente (2007) MMA divulga lista dos municípios que mais desmataram em 2007. Disponível em: <https://www.mma.gov.br/informma/item/4590mmadivulga-lista-dos-municipios-que-maisdesmataram-em-2007 >Acesso 16 julho 2020. 
Petini-Benelli A, Lopes CRAS, Silva DR, Ribeiro RS (2014) Novos registros de epífitas vasculares para o estado de Mato Grosso, Brasil. 11: 23402351.

Petini-Benelli A, Soares-Lopes CRA (2015) A new species of Catasetum (Cymbidieae, Epidendroideae, Orchidaceae) from the Southern region of the Brazilian Amazon. Phytotaxa, 204: 75-79.

Petini-Benelli A (2016) Un novel hybride naturel de Catasetum (Cymbidieae, Epidendroideae, Orchidaceae) du Mato Grosso (Brésil). Richardiana, 26: 327-342.

Petini-Benelli A, Soares-Lopes, CRA (2017) New taxa of Catasetum (Orchidaceae, Catasetinae) from Mato Grosso, Brazil. Richardiana, 1: 31-43.

Petini-Benelli, Izzo TJ (2017) Catasetum brasilandense (Orchidaceae), a new species from Mato Grosso, Brazil. Richardiana, 1: 51-62.

RADAMBRASIL (1978) Folha SC.21 - Juruena. Rio de Janeiro: Radambrasil, 117-164.

Ribeiro RS, Almeida, AASD, Bortolan G B, Rodrigues L, Silva DR, Sá CF, Santos, PS, Lopes CRAS (2016) Verbenaceae J.St.-Hil (Lamiales) depositadas no acervo do Herbário da Amazônia Meridional - HERBAM. Scientific Electronic Archives, 9: 377 - 379. doi: 10.36560/962016470

Ribeiro RS, Soares-Lopes CRA (2016) Biogeografia das Bignonieae (Bignoniaceae, Lamiales) ocorrentes no município de Nova Bandeirantes, MT: diminuindo lacunas e fornecendo dados para a biologia da conservação. In. IX Semana da Biologia, Alta Floresta.

Ribeiro RS, Almeida AASD, Cardoso SMC, Koch AK, Soares-Lopes CRA (2018a) Bignoniaceae (Lamiales) ocorrentes no Município de Jacareacanga, Amazônia Meridional (Pará, Brasil). Scientific Electronic Archives, 11: 409-413.

Ribeiro RS, Lohmann LG, Lopes CRAS (2018b) Espécies da "Aliança Tabebuia" (Bignoniaceae, Lamiales) ocorrentes no município de Alta Floresta, Mato Grosso. In: VII Simpósio da Amazônia Meridional em Ciências Ambientais, Sinop.

Ribeiro RS, Lohmann LG, Soares-Lopes CRAS (2018c) Estudos taxonômicos da Tribo Bignonieae (Bignoniaceae, Lamiales) no município de Alta Floresta (Mato Grosso, Brasil). In: $69^{\circ}$ Congresso Nacional de Botânica, Cuiabá.

Ribeiro RS, Soares-Lopes CRA (2018) Flora de Angiospermas do município de Nova Bandeirantes,
Mato Grosso (Brasil): Bignoniaceae Juss. Scientific Electronic Archives, 11: 84.

Ribeiro RS (2018) Checklist da família Bignoniaceae Juss. (Lamiales) ocorrentes no Município de Alta Floresta, Mato Grosso, Brasil. Trabalho de Conclusão de Curso. Universidade do Estado de Mato Grosso, 72p.

Ribeiro RS, Soares-Lopes CRA (2018) Status atual da Flora Matogrossense revelada via GBIF (Biodiversity Information Facility). Scientific Electronic Archives 11: 83.

Ribeiro RS, Pereira NV, Cardoso SMC, Almeida AASD, Pasa, MC (2020) Usando banco de dados online das coleções botânicas para sintetizar o conhecimento taxonômico e geográfico atual da flora e fungos no estado de Rondônia (Brasil). Biodiversidade, 9: 34-43.

Ribeiro RS, Lohmann LG, Lopes CRAS (2021) Flora of the State of Mato Grosso, Brazil: Bignoniaceae of Mato Grosso. [Field Guides, Field Museum] Field Museum, 10p. Disponível em: https://fieldguides.fieldmuseum.org/guides/guide/1 333 Acesso em 27 de Junho de 2021.

Shepherd, GJ (2003) Avaliação do estado do conhecimento da diversidade biológica do Brasil: Plantas terrestres (versão preliminar). Ministério do Meio Ambiente/Secretaria de Biodiversidade e Florestas, Brasília. Disponível em: http//:www.mma.gov.br/estruturas/chm/arquivos/p lantas1.pdf Acesso em 15 de Julho de 2020.

Silva DR, Soares-Lopes CRA, Verão DS, Rodrigues L, Ribeiro RS (2015) Loranthaceae Juss. no Acervo do no acervo do Herbário da Amazônia Meridional, Alta Floresta, Mato Grosso. Enciclopédia Biosfera, 11: $3690-3702$.

Silva DR, Soares-Lopes CRA, Gressler E, Eisenlohr PV (2020) Woody vegetation associated with rocky outcrops in Southern Amazonia: a starting point to unveil a unique flora. Biota Neotropica, 20: p.e 20190874

Silva AS, Fernandes JM, Soares-Lopes, CRA (2019) Taxonomia do gênero Albizia (Leguminosae) no estado de Mato Grosso, Brasil. Enciclopédia Biosfera 16: 1-14.

Soares-Lopes CRA, Ribeiro RS, Silva DR, Verão DS, Rodrigues, L. Oliveira CN, Spletozer AG, Santos CR, Santos LG, Lopes FJA, Almeida AASD, Sá CF, Del Rei M, Piva J.H, Sousa L, Rodrigues C, Mello JM, José Fernandes JM, Koch AK, PetiniBenelli A, Silveira M (2016a) Enclaves de Cerrado S.S. Na Amazônia Mato-Grossense: Uma Proposta 
Fitogeográfica. Scientific Electronic Archives, 9: 468-471.

Soares-Lopes CRA, Silva DR, Ribeiro RS, Verão DS, Rodrigues, L. Oliveira CN, Spletozer AG, Santos CR, Santos LG, Lopes FJA, Almeida AASD, Sá CF, Del Rei M, Piva J.H, Sousa L, Rodrigues C, Mello JM, José Fernandes JM, Koch AK, PetiniBenelli A, Silveira M (2016b) Composição Florística de um Enclave de Cerrado S.S. Na Amazônia Mato-Grossense: Dados Preliminares. Scientific Electronic Archives, 9: 472-474.

Souza SD, Spletozer AG, Rodrigues L, SoaresLopes, CRA (2016) Contrivuição taxonômica ao estudo do gênero Senna Mill. (Leguminosae: Caesalpinoideae) no estado de Mato Grosso, Brasil. Enciclopédia Biosfera, 13: 322-330.

Teixeira FC, Ribeiro RS, Spletozer AG, Almeida AASD, Rodrigues L, Silva DR, Da Silva, DF, Lopes, CRAS (2016) Checklist e distribuição geográfica das Acanthaceae Juss. (Lamiales) da Amazônia Norte Mato-Grossense. Scientific Electronic Archives, 9: p.490 - 492, 2016.

ter Steege H, Mota de Oliveira S, Pitman NCA, Sabatier D, Antonelli A, Andino JEG, Aymard GA, Salomão RP (2019) Towards a dynamic list of Amazonian tree species. Scientific Reports, 9(3501): 1-5. doi: 10.1038/s41598-019-40101-y

Thiers B (2020) Index Herbariorum: A Global Directory of Public Herbaria and Associated Staff. New York Botanical Garden's Virtual Herbarium. 2020. Disponível em: <http://sweetgum.nybg.org/science/ih/> Acesso Julho de 2020.

Thomas WW, Forzza RC, Michelangeli FA, Giulietti AM, Leitman PM (2012_) Large-scale monographs and floras: the sum of local floristic research. Plant Ecology \& Diversity, 5(2): 217-223. doi: 10.1080/17550874.2011.622306

Ulloa Ulloa C, Acevedo-Rodríguez P, Beck S, Belgrano MJ, Bernal R, Berry PE, Brako L, Celis M, Davidse G, Forzza RC, Gradstein SR, Hokche O, Léon B, León-Yánez S, Magill RE, Neill DA, Nee M, Raven PH, Stimmel H, Strong MT, Villaseñor JL, Zarucchi JL, Zuloaga FO \& Jørgensen PM (2017) An integrated assessment of the vascular plant species of the Americas. Science 358: 16141617. DOI: $10.1126 /$ science.aao0398.

Zappi DC, Sasaki D, Milliken W, Piva J, Henicka GS, Biggs N, Frisby S (2011) Plantas vasculares da região do Parque Estadual Cristalino, norte de Mato Grosso, Brasil. Acta Amazonica 41: 29-38 doi: $10.1590 /$ S0044-596720
Zappi DC, Milliken W, Lopes CRAS, Lucas E, Piva JH, Frisby S, Biggs N \& Forzza RC (2016) Xingu State Park vascular plant survey: filling the gaps. Brazilian Journal of Botany 39: 751-778. 